\title{
Chromosomes and Karyotype of the Indian Pangolin, Manis crassicaudata Gray (Pholidota-Mammalia)
}

\author{
N. V. Aswathanarayana* \\ University of Mysore, Manasa Gangotri, Mysore-570 006, India \\ Accepted August 9, 2000
}

\begin{abstract}
Summary The chromosomes and the karyotype of a female pangolin, Manis crassicaudata collected from Mysore (south Inida) have been described. The karyotype consists of a diploid number of 36 chromosomes. All the autosomes are biarmed except one small and telocentric pair with 7 pairs of large to small metacentric, 6 pairs of submetacentric and 3 pairs of large subtelocentric chromosomes. The $\mathrm{X}$ chromosome is a medium sized submetacentric. This study has shown that the pangolins of Varanasi (north India) and Bellary (south India) belong to M. crassicaudata and not $M$. pentadactyla and thus widened the horizon for further karyological studies of pangolins.
\end{abstract}

The Pangolins or scaly anteaters of the old world come under Order Pholidota which has only one family, Manidae and one genus, Manis (Prater 1971, Corbet and Hill 1992). The chromosome information of pangolins is very meager. The first report on this group is of Makino and Tateishi in 1951, (see Ray-Chaudhuri et al. 1969) who reported the chromosomes of a male pangolin, Manis pentadactyla from Japan with $2 \mathrm{n}=42$. Later, Ray-Chaudhuri et al. (1969) described a karyotype with $2 n=36$ in a male specimen of the same species procured from a forest area, 30 miles away from Varanasi (Uttar Pradesh, North India) using the usual air-dry technique. Satya-Prakash and Aswathanarayana (1972) reported $2 n=36$ in a female pangolin of the same species from Bellary, (Karnataka, south India). Chakrabarti et al. (1982) presented a detailed account of the karyotypes of both sexes of $M$. pentadactyla, having $2 \mathrm{n}=40$ from the environs of Bengal and Tripura (North-east Indian states). Because of 3 chromosomes numbers of $2 n=42,40$ and 36 for the same species, $M$. pentadactyla, it was thought relevant to know whether the above said pangolins belong to same species or different species. In this context the literature on the taxonomy and distribution of pangolins was referred from which it became clear that there are 2 distinct species of pangolins in India, Manis crassicaudata Gray and M. pentadactyla Linn. based on morphological characters like body measurements, tail and distribution of scales (Table 1). M. crassicaudata is the common Indian pangolin and is distributed in Pakistan, peninsular India, N.E. to Bengal, Bangladesh and Sri Lanka. M. pantadactyla is the Chinese pangolin whose distribution ranged westwards through Assam and eastern Himalayas to Nepal, Burma and south China (Prater 1971, Corbet and Hill 1992). So the pangolin species available in south india must be $M$. crassicaudata. The name $M$. pentadactyla was used in the past for the Indian pangolin, M. crassicuadata.

Now the author is reporting the chromosomes and karyotype of the Indian pangolin, M. crassicuadata and compares with other karyotypes to solve the controversy regarding the diploid number and the identification of the species studied.

\section{Material and methods}

One female pangolin, Manis crassicaudata collected in Mysore was used in this study, (Karnataka, south India). The routine air-dry technique was applied for chromosome preparations using

* Present Address: 290/3, 34 'A' Cross, 9th Main, IVth Block, Jayanagar, Bangalore-560 011, India. 
Table 1. Morphological features of the species of Manis

\begin{tabular}{|c|c|c|}
\hline & pentadactyla $(\mathrm{cm})$ & crassicaudata $(\mathrm{cm})$ \\
\hline Head and body & $48-58$ & $60-75$ \\
\hline Tail & $33-38$ & 45 \\
\hline Rows of scales around mid-body & $15-18$ & $11-13$ \\
\hline Mid-dorsal scales (posterior margin) & Most truncate & Most convex \\
\hline Scales along edge of tail & $16-19$ & $14-15$ \\
\hline Terminal scale under tail & Membranous & Hard \\
\hline
\end{tabular}

bone marrow and spleen and stained with Giemsa. Bone marrow cells yielded fairly good metaphases. Banding was induced by applying the technique of Sumner (1972) with slight modification.

\section{Observations}

Fifty well spread metaphases showed a diploid number of 36 chromosomes with XX sex mechanism (Fig. 1). All the chromosomes are biarmed except one pair which is the smallest and telocentric. The autosomes are categorised into 7 pairs of small to large metacentric (1-7), 6 pairs of submetacentric (8-13) which are in a graded series and 3 pairs of comparatively large subtelocentric chromosomes (14-16). A medium sized submetacentric is tentatively identified as the $\mathrm{X}$ chromosome. Banding preparations were not satisfactory for presentation.

\section{Discussion}

At present there are 4 studies on the chromosomes of pangolin, Manis pentadactyla. (1) In 1951, Makino and Tateishi (Ray-Chaudhuri et al.1969) reported a diploid number of 42 in a male pangolin from Japan. Since they used paraffin section method the Japanese material has to identified and restudied to confirm their observation. (2) Ray-Chaudhuri et al. (1969) described $2 n=36$ in the male collected from Varanasi (Uttar Pradesh) giving the metaphase, the karyotype and the idiogram. Their karyotype has 16 pairs of biarmed autosomes of which 7 pairs are metacentric (small to large), 6 pairs are submetacentric and 3 pairs of large sized subtelocentric chromosomes. The last pair is the smallest autosome and acrocentric. The $\mathrm{X}$ chromosome is a medium sized submetacentric and $\mathrm{Y}$ is an acrocentric chromosome. (3) Satya-Prakash and Aswathanarayana (1972) also reported a karyotype with $2 n=36$ in a female specimen procured from Bellary (Karnataka) having 7 pairs of metaceantric, 6 pairs of submetacentric 3 pairs of subtelocentric and one pair of telocentric autosomes. One pair of submetacentric chromosomes is tentatively identified as the $\mathrm{X}$ chromosomes. (4) Chakrabarti et al. (1982) have presented the karyotypes of both sexes and C-banding for the chromosomes of pangolin collected from Bengal and Tripura states. They describe $2 n=40$ for the species. Their karyotype has 38 biarmed autosomes consisting of 5 pairs of metacentrics, 6 


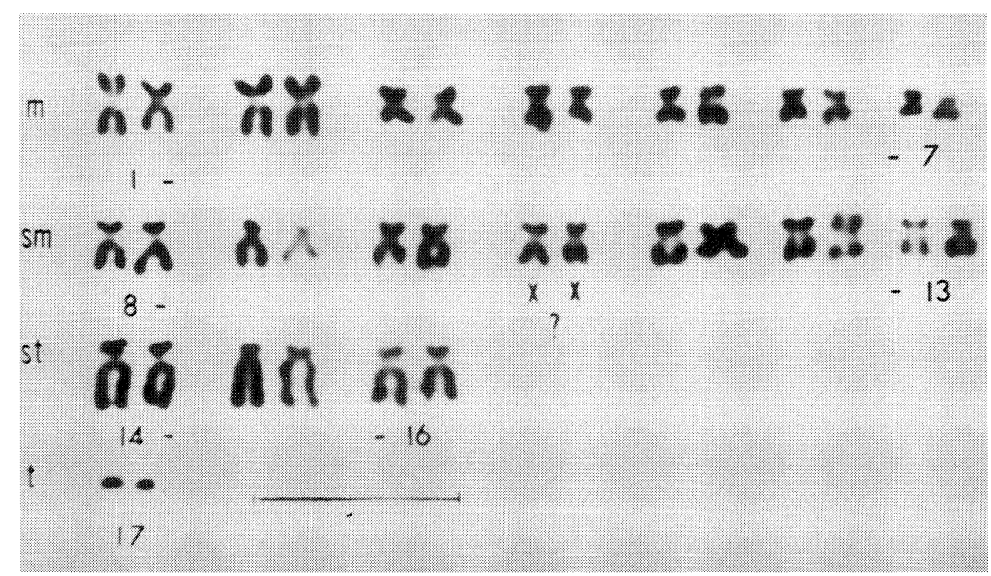

Fig. 2. Karyotype of the female M. crassicaudata. Bar scale $10 \mu \mathrm{m}$.

Table 2. Comparison of the Chromosomes of the species identified as Manis pentadactyla

\begin{tabular}{lccccccccc}
\hline \multicolumn{1}{c}{ Authors } & 2n & M & SM & ST & T/Acro & X & Y & Sex \\
\hline Ray-Chaudhuri et al. (1969) & 36 & 14 & 12 & 6 & 2 & sm & acro & male \\
Satya-Prakash and Aswathanarayana (1972) & 36 & 14 & 12 & 6 & 2 & sm & $?$ & $\begin{array}{c}\text { female } \\
\text { both sexes } \\
\text { Chakrabarti et al. (1982)* }\end{array}$ \\
\hline
\end{tabular}

* Last 4 chromosomes bear satellites.

pairs of submetacentric, 7 pairs of subtelocentric (small to large) and one pair of telocentric chromosomes. The $\mathrm{X}$ chromosome is a medium sized metacentric and $\mathrm{Y}$ is a small submetacentric element. In the C-banding $\mathrm{X}$ chromosome has a dark centromeric band and $\mathrm{Y}$ is completely heterochromatic. They also report that the last 4 chromosome bear satellites in their short arms. Their karyotype is constructed based on chromosome length and they have not catagorised into distinct groups with description which becomes problematic when other karyotypes are campared. However this karyotype has some distinct differences with others in the number and morphology of the chromosomes which are tabulated (Table 2).

The chromosome number in $M$. crassicaudata of the present study is also $2 n=36$. All the chromosomes are biarmed except the last pair which is the smallest and telocentric. The biarmed autosomes include 7 pairs of large to small metacentric, 6 pairs of medium sized submetacentric, 3 pairs of subtelocentric, and one pair of small telocentric chromosomes. The $\mathrm{X}$ chromosome is difficult to identify in the female. However, by comparing the male karyotype described by Ray-Chaudhuri et al. (1969) which has also $2 \mathrm{n}=36$, a medium sized submetacentric is identified as the X chromosome. The present karyotype has no SAT chromosomes. The C-band preparations were not satisfactory for presentation. The attempt to use another specimen was given up as pangolin is a precious species.

The architecture of the present karyotype of $M$. crassicaudata conforms to the karyotype presented by Ray-Chaudhuri et al. (1969) and Satya-Prakash and Aswathanarayana (1972). Slight differences if any in the morphology of chromosomes can be attributed to differential condensation. The difference between this karyotype and that described by Chakrabarti et al. (1982) are same as in Table 2. However, the common features of the both the Karyotypes of $2 n=40$ and $2 n=36$ are the presence of biarmed chromosomes and acro/telocentric nature of the last pair of chromosomes. Because of this one can not say which karyotype is more advanced. 
Since the karyotypes of pangolin described by Ray-Chaudhuri et al. (1969) and Satya-prakash and Aswathanarayana (1972) with 2n=36 from north india (Vasanasi) and south India (Bellary) respectively are so similar to that of the present $M$. crassicaudata (identified based on morphological characters) they definitely belong to $M$. crassicaudata and not $M$. pentadatyla. Therefore, it is clear that there are 2 distinct species in India, $M$. crassicaudata with $2 \mathrm{n}=36$ and $M$. pentadactyla with $2 n=40$. This study has thus puts an end to the controversy of the diploid number and identification of the 2 species of the genus Manis.

\section{Acknowledgment}

The author is highly thankful to Dr. Ashok Kumar Singh of the Zoological Survey of India, Calcutta for making available the relevant literature on taxonomy and distribution of pangolins.

\section{References}

Chakrabarti, S., Bhattacharyya, S. and Chakarabarti, A. 1982. Somatic chromosomes of pangolin, Manis pentadactyla (Pholidota; Mammalia) with special reference to satellite association. Cytologia 47: 625-629.

Corbet, G. B. and Hill, J. E. 1992. The Mammals of the Indomalayan Region. In: A Systematic Review, Natural History Museum Publ. Oxford Univ. Press. pp. 1-488.

Prater, S. H. 1971. Pangolins. In: The Book of Indian Animals, Bombay Natural History Society and Oxford Univ. Press, Bombay. pp. 1-324.

Ray-Chaudhuri, S. P., Sharma, T., Pathak, S. and Singh, L. 1969. Chromosomes and the karyotype of pangolin, Manis pentadactyla L. (Pholidota-Mammalia). Experientia 25: 1167-1168.

Satya-Prakash, K. L. and Aswathanarayana, N. V. 1972. Karyotype of the female pangolin, Manis pentadactyla L. (Pholidota-Mammalia) Mamm. Chrom. Newsl. 13: 142-143.

Sumner, A. T. 1972. A simple technique for determining centromeric heterochromatin. Exp. Cell Res. 75: 304-306. 